\title{
On some peculiar aspects of the constructive theory of point-free spaces
}

\author{
Giovanni Curi \\ Dipartimento di Informatica - Università di Verona \\ Strada le Grazie 15 - 37134 Verona, Italy. \\ e-mail: giovanni.curi@univr.it
}

July $21,2009^{*}$

\begin{abstract}
This paper presents several independence results concerning the topos-valid and the intuitionistic (generalized) predicative theories of locales. In particular, certain consequences of the consistency of a general form of Troelstra's uniformity principle with constructive set theory and type theory are examined.
\end{abstract}

\section{Introduction}

It may be argued that the well-known equivalence of theorems such as Tychonoff theorem, or Stone-Čech compactification, with the axiom of choice, or other similarly non-constructive principles, is not an intrinsic feature of these results, but, rather, is determined by the chosen formulation of the concept of space. By replacing the ordinary notion of topological space with that of locale (or frame, or complete Heyting algebra) one obtains fully general versions of these theorems that can be proved without any choice, and often with no application of the principle of excluded middle [22, 16, 15, 21].

The notion of locale is for this reason the concept of space generally adopted in choice-free intuitionistic settings, such as toposes or intuitionistic set theory (IZF) 28. By not assuming as available impredicative principles as the existence of powersets, the concept of formal space, or set-generated locale, plays a corresponding role in even weaker systems, as constructive set theory (CZF) or constructive type theory (CTT) [32, 10, 1, 26, 12]. The main criterion of adequateness of this notion

${ }^{*}$ First version: February 1, 2006. 
is that, considered in fully impredicative settings, the category FSp of formal spaces is equivalent to the ordinary category of locales.

This paper deals with certain peculiar features of both the constructive and the intuitionistic theory of locales. In fact, we will mostly be concerned with certain independence results that follow from the consistency of CZF and CTT with a generalized form of Troelstra's principle of uniformity [35].

Our main results are related to the following fundamental 'structural' aspect of the theory of locales: considered in any topos, the category of locales is complete and cocomplete, i.e. all limits (in particular products) and colimits exist in this category. By contrast, already the existence of binary products of arbitrary formal spaces seems to require the use of strongly impredicative principles, not available in the generalized predicative settings under consideration. In particular to remedy this deficiency, the concept of inductively generated formal space has been introduced in [10, 1]: inductively generated formal spaces define a full subcategory $\mathrm{FSp}_{i}$ of the category of formal spaces in which limits and colimits do exist (although under the assumption of strong principles for the existence of inductively defined sets, e.g. the axiom REA in constructive set theory, see e.g. [26, 1]).

By exhibiting a particular formal space that CTT cannot prove to be inductively generated, $\mathrm{FSp}_{i}$ has been shown to form a proper subcategory of FSp in [10] (see [17. for a similar result in $\mathrm{CZF}$ ). Nevertheless, since $\mathrm{FSp}_{i}$ contains in particular all locally compact formal spaces, and since, considered in a fully impredicative setting, this category is still equivalent to the category of locales (as every formal space is inductively generated in such a setting), the concept of inductively generated formal space has generally been regarded as providing the proper constructive analogue of the notion of locale.

Our main results will show that the restriction to the category $\mathrm{FSp}_{i}$ is however a very severe one: we show that CTT, CZF, as several extensions of CZF, including REA and the impredicative unbounded Separation Scheme, cannot prove that a non-trivial Boolean formal space - i.e. a formal space whose associated frame is a non-trivial complete Boolean algebra -, is inductively generated. This result provides us, in particular, with an example of a formal space that these systems cannot prove to be inductively generated for every given formal space (inductively generated or not), namely its least dense subspace. Similar facts also hold for De Morgan (or extremally disconnected) formal spaces, and for formal spaces whose associated frame is the the Dedekind-MacNeille completion of a poset.

Further independence results, concerning compactness, overtness (openness) and existence of points, are also shown to hold with respect to the internal language of toposes (HHA), IZF, and/or CTT and CZF. In particular, we show that CZF (+REA), CTT cannot prove that a formal space is compact and De Morgan. This is in contrast with a well-known topos-valid result of M. Stone, by which the frame 
of ideals of a complete Boolean algebra is a compact De Morgan locale.

The paper is organized as follows: basic facts on formal spaces/locales as treated in constructive settings are recalled in Section 0, In Section 10 the version of the uniformity principle we shall exploit is presented and its incompatibility with De Morgan law is exhibited. The independence results concerning Boolean and De Morgan formal spaces are described in Section 2, the case of spaces arising via the Dedekind-MacNeille completion of a poset, and a problem left open in [10, are discussed in Section 3 .

\section{Preliminaries}

The reader is referred to [3, 29] for background on Aczel's constructive set theory and constructive type theory (CTT), respectively. In the following, we shall use CZF to strictly indicate the basic formulation of constructive set theory. An often considered extension of CZF, particularly in connection with constructive locale theory, is the theory CZF+REA. The regular extension axiom REA is needed to ensure that certain inductively defined classes are sets [3]. With HHA (intuitionistic higher-order Heyting arithmetic) we shall indicate topos logic [28, 35. General information on locales may be found in [15, 16, 21]; for basic facts concerning the theory of formal spaces in constructive predicative settings such as CZF, CTT the reader may consult $[1,10,32,26$, or $[2,12$. Here we synthetically recall the notions needed in this note.

A formal topology, or formal space, is a pair $S \equiv(S, \triangleleft)$ where $S$, the base, is a set, and $\triangleleft$, the covering relation, is a relation between elements and subsets of $S$ satisfying:

i. $a \in U$ implies $a \triangleleft U$,

ii. if $a \triangleleft U$ and $U \triangleleft V$, then $a \triangleleft V$,

iii. $a \triangleleft U$ and $a \triangleleft V$ imply $a \triangleleft U \downarrow V$,

where $U \triangleleft V \equiv(\forall u \in U) u \triangleleft V$, and $U \downarrow V \equiv\{d \in S:(\exists u \in U)(d \triangleleft\{u\}) \&(\exists v \in$ $V)(d \triangleleft\{v\})\}$. Being contained in $S \times \operatorname{Pow}(S)$, the covering is a class-relation in $\mathrm{CZF}$; a further requirement in that context is that the class $\mathcal{S}(U) \equiv\{a: a \triangleleft U\}$ is a set for all $U$ (see [1] for more). Two subsets $U, V$ of $S$ are the same formal open, $U={ }_{S} V$, exactly when $U \triangleleft V \& V \triangleleft U$. Observe that one may always assume $S$ to have a top element, i.e. an element $1_{S}$ such that $S={ }_{S}\left\{1_{S}\right\}$. An implication operation is defined on formal opens by letting $U \rightarrow V \equiv\{a \in S: a \downarrow U \triangleleft V\}$. The pseudocomplement $U^{*}$ of $U$ (the largest open disjoint from $U$ ) is given by:

$$
U^{*} \equiv U \rightarrow \emptyset \equiv\{a \in S: a \downarrow U \triangleleft \emptyset\} .
$$


A morphism $f: S_{1} \rightarrow S_{2}$ of formal topologies is a mapping $f: S_{1} \rightarrow \operatorname{Pow}\left(S_{2}\right)$ satisfying

$$
\begin{aligned}
& \text { i. } f\left(S_{1}\right)=S_{2} S_{2}, \\
& \text { ii. } f(a) \downarrow f(b) \triangleleft f(a \downarrow b), \\
& \text { iii. } a \triangleleft U \rightarrow f(a) \triangleleft f(U),
\end{aligned}
$$

where, for $U$ a subset of $S_{1}, f(U) \equiv \bigcup_{a \in U} f(a)$. Two morphisms $f, g$ are defined to be equal precisely when $f(a)=S_{2} g(a)$ for all $a \in S_{1}$. On any formal topology $S$, the identity morphism is given by $i d_{S}(a)=\{a\}$, for all $a$.

A (formal) point of a formal space $S$ is a subset $\alpha$ of $S$ satisfying:

i. $(\exists a \in S) a \in \alpha$

ii. $a, b \in \alpha$ implies $(\exists c \in \alpha) c \in a \downarrow b$.

iii. $a \in \alpha$ and $a \triangleleft U$ imply $(\exists b \in U) b \in \alpha$.

In particular, the top element (when it is given) belongs to every point, and for no $a \in \alpha, a \triangleleft \emptyset$.

Even classically, a formal space may well have no points and be non-trivial. In terms of logic, this is because points of a frame are the Pow $(\{\top\})$-valued (classically two-valued) models of a geometric theory, which may be consistent without having a model [15].

A subspace of a formal space $S \equiv(S, \triangleleft)$ is a formal space $S^{\prime} \equiv\left(S, \triangleleft^{\prime}\right)$, on the same base, and with $\triangleleft^{\prime}$ satisfying $i . \triangleleft \subseteq \triangleleft^{\prime}$, and ii. $x \downarrow^{\prime} y \triangleleft^{\prime} x \downarrow y$. Two subspaces $S^{\prime}, S^{\prime \prime}$ are equal if $a \triangleleft^{\prime} U \Longleftrightarrow a \triangleleft^{\prime \prime} U$. See [12] for more. For example, a (formal) open subset $V \subseteq S$ determines the closed subspace $S^{V} \equiv\left(S, \triangleleft^{V}\right)$, with $a \triangleleft^{V} U \Longleftrightarrow a \triangleleft U \cup V$ (intuitively, $S^{V}$ represents the complement of the open $V$ as a subspace).

A formal space $S$ is set-presented iff there are families of sets $I(x)$ for $x$ in $S$ and $C(x, i) \subseteq S$, for $x \in S, i \in I(x)$, such that

$$
a \triangleleft U \Longleftrightarrow \exists i \in I(a) C(a, i) \subseteq U .
$$

Observe that this implies $a \triangleleft C(a, i)$ for all $i$. In CZF+REA, CTT, $S$ is setpresented if and only if it is inductively generated in the sense of [1, 10]. In CZF, CTT, the class of set-presented formal spaces contains all locally compact spaces [1, 11. In a topos, or in (I)ZF, all formal spaces are trivially set-presented: one 
simply defines $I(x)=\{U \in \operatorname{Pow}(S): x \triangleleft U\}, C(x, U)=U$. The full subcategory of set-presented formal spaces has limits and colimits in sufficiently strong versions of constructive set theory and type theory.

In CZF, a class-frame (or class-locale) $L$ is a partially ordered class that has a top element, binary meets, and suprema for arbitrary sets of elements of $L$ that distribute over meets. A class-frame is said to be set-generated by a subclass $B$ if: $i$. $B$ is a set, $i i$. the class $\{b \in B: b \leq x\}$ is a set and $x=\bigvee\{b \in B: b \leq x\}$, for all $x \in L$.

Morphisms of set-generated frames are class-functions respecting meets, the top, and arbitrary joins.

Given a formal topology $S$, let $\operatorname{Sat}(\mathcal{S})$ denote the collection of saturated subsets of $S$, i.e. the class $\{U \subseteq S: \mathcal{S}(U)=U\}$. Endowed with the operations $U \wedge V \equiv$ $U \cap V=U \downarrow V$ and $\bigvee_{i \in I} U_{i} \equiv \mathcal{S}\left(\bigcup_{i \in I} U_{i}\right)$, Sat $(S)$ is a set-generated frame. The implication operation previously recalled defines an implication operation on $\operatorname{Sat}(\mathcal{S})$ in the usual sense, making it in a complete Heyting algebra. In particular, $U^{*}$, for $U \in \operatorname{Sat}(S)$ is the pseudocomplement of $U$ in the sense of Heyting algebras.

With their respective morphisms, formal topologies and set-generated classframes form equivalent categories [1, 2]. With powersets, every set-generated classframe has a set of elements, so it is just an ordinary frame (locale). Therefore, in fully impredicative settings such as toposes, the category FT of formal topologies is equivalent to that of frames (see also [32]). Its opposite $\mathbf{F S p}=\mathbf{F} \mathbf{T}^{o p}$, here referred to as the category of formal spaces (often simply spaces) and continuous functions, is thus equivalent in such settings to the category of locales.

\section{Uniformity principles}

To distinguish the behavior of formal spaces in constructive settings from that in an intuitionistic but fully impredicative context, a generalized form of the so called uniformity principle [35, 31] will be exploited. In constructive set theory this takes the following form: for every set $I$,

$$
(\forall x)(\exists y \in I) A(x, y) \rightarrow(\exists y \in I)(\forall x) A(x, y) \quad \text { (GUP-CZF). }
$$

This principle has been proved to be consistent (in particular) with CZF+REA+ PA+Sep in [4, 5] (see also [27, 34]), where REA is the regular extension axiom, PA is the presentation axiom, and Sep is impredicative unbounded separation. Note that GUP-CZF follows from its instance:

$$
(\forall x)(\exists y \in \omega) A(x, y) \rightarrow(\exists y \in \omega)(\forall x) A(x, y) \quad(\mathbf{U P}-\mathbf{C Z F})
$$

( $\omega$ is the set of natural numbers), and the principle that every set is subcountable, also valid in the model of GUP described in [5]. 
It will be convenient to note explicitly the following consequence of GUP-CZF: for every set $I$,

$$
(\forall p \in \operatorname{Pow}(\{\top\}))(\exists i \in I) A(p, i) \rightarrow(\exists i \in I)(\forall p \in \operatorname{Pow}(\{\top\})) A(p, i)
$$

where $\operatorname{Pow}(\{\top\})$ is the powerclass of the one-element set (the antecedent of GUP'CZF yields $(\forall x)(\exists y \in I)(\exists z)(z=x \cap\{\top\} \& A(z, y))$; one can then apply GUP-CZF). The type-theoretic formulation of this principle, first exploited in [10], is recalled in the Appendix.

Recall that De Morgan law $\neg(P \wedge Q) \rightarrow \neg P \vee \neg Q$ for all propositions $P, Q$, is equivalent to

$$
\neg \neg P \vee \neg P \quad(\mathbf{D M L})
$$

for all $P$. By the identification of subsets of the one-element set with restricted formulas (those in which quantifiers appear only bounded) [3], in CZF this principle for restricted formulas can be formulated as

$$
(\forall p \in \operatorname{Pow}(\{\top\})) p^{* *} \cup p^{*}=\{\top\} \quad([\mathbf{R}] \mathbf{D M L})
$$

where $p^{*}$ is the set-theoretic complement of $p$. Note that the last formula considered in IZF expresses De Morgan Law for arbitrary formulas.

The generalized uniformity principle conflicts with DML ([R]DML). We prove this in CZF: assume that $p^{* *} \cup p^{*}=\{\top\}$ for all $p \in \operatorname{Pow}(\{\top\})$. Define a relation $F \subseteq \operatorname{Pow}(\{\top\}) \times\{0,1\}$ by letting

$$
(x, y) \in F \Longleftrightarrow\left(\top \in x^{* *} \& y=1\right) \vee\left(\top \in x^{*} \& y=0\right) .
$$

By the assumption one has $\left(T \in x^{* *}\right) \vee\left(T \in x^{*}\right)$ for all $x \in \operatorname{Pow}(\{\top\})$, so that trivially

$$
(\forall x \in \operatorname{Pow}(\{\top\}))(\exists y \in\{0,1\})(x, y) \in F .
$$

By GUP-CZF, this gives

$$
(\exists y \in\{0,1\})(\forall x \in \operatorname{Pow}(\{\top\}))(x, y) \in F,
$$

which yields a contradiction (consider $x=\{\top\}, x=\emptyset$, for $y=0, y=1$, respectively).

Of course, this implies that GUP is inconsistent with the principle of excluded middle PEM in CTT, in CZF with excluded middle for restricted formulas, or equivalently, with

$$
(\forall p \in \operatorname{Pow}(\{\top\})) p \cup p^{*}=\{\top\} \quad([\mathbf{R}] \mathbf{E M})
$$


(again, considered in IZF, [R]EM is equivalent to the full law of excluded middle).

In the following, we shall for simplicity write 'constructive settings' to indicate generically CTT, CZF, or any of their extensions which are compatible with the generalized uniformity principle (so the terminology is quite improper, given that CZF plus impredicative unbounded separation is one such extension). With 'intuitionistic setting' we indicate any of CTT, CZF, IZF, HHA.

We will freely use the fact that all the settings in consideration may consistently be extended with the negation of the law of (restricted) excluded middle (set-theoretically with $\neg \forall p \in \operatorname{Pow}(\{\top\}) p \cup p^{*}=\{\top\}$ ). The assertion that a space of a certain type cannot be proved to have a certain property in a certain setting will invariably be proved by showing that in the setting extended with some compatible non-classical principle (as GUP, or $\neg$ PEM), the assumption that the considered space has the property is contradictory.

The proofs are carried out in the setting of constructive set theory. The reader should have no difficulty to modify them according to the setting he is more familiar with.

\section{Boolean and De Morgan locales/formal spaces}

By exploiting the generalized uniformity principle it is shown in [10] that there is a formal space that CTT cannot prove to be set-presented; in [18] it is shown by other means that the system CZF cannot prove the so-called 'double-negation' formal space (see Section [3) to be set-presented1 1 . We shall see in Section 3 that these spaces are in fact isomorphic, and that their associated frame is a complete Boolean algebra. The same argument given in [18] seems then to show that any formal space whose associated frame is a non-trivial (complete) Boolean algebra cannot be proved to be set-presented over the basic set of axioms of CZF2.

In this section, using the generalized uniformity principle we give a simple proof that the systems $\mathrm{CTT}$ and $\mathrm{CZF}+\mathrm{REA}+\mathrm{PA}+\mathrm{Sep}$ cannot prove a non-trivial Boolean formal space to be set-presented. A similar result is shown to hold also for De Morgan (or extremally disconnected) formal spaces. In fact, these results hold true for any extension of (a fragment of) CTT or CZF compatible with the generalized uniformity principle (but we shall focus on the indicated ones; see [5] for a list of other principles compatible with GUP).

Further independence results concerning overtness, compactness and existence of points are also obtained. Aside from Theorem [2.9, these make no use of the

\footnotetext{
${ }^{1}$ As noted in [18, R. Grayson [19] had obtained a corresponding result for certain formulations of intuitionistic set theory without Powerset.

${ }^{2}$ This observation is essentially due to S. Vickers; in fact also the corresponding of this result was known to Grayson [19] in connection with the set theories he considered (cf. footnote 1).
} 
consistency of GUP with the given setting and hold true, mutatis mutandis, also with respect to topos logic (HHA), or IZF. All results in which the generalized uniformity principle is involved, which thus only concern the constructive settings, will be marked with GUP.

Call a formal space $S$ such that $\operatorname{Sat}(S)$ is a Boolean frame a Boolean formal space. From now on, let for simplicity $S$ have a top basic element $1_{S}$. If $S$ is setpresented, also the enlargement of its base with a top element $1_{S}$ can be proved to be set-presented (this is proved in type theory using type-theoretic choice [12], in constructive set theory exploiting the Subset Collection scheme). Thus, a Boolean formal space is one such that $1_{S}={ }_{S} U \cup U^{*}$ for all $U \in \operatorname{Pow}(S)$.

A formal space $S$ is De Morgan if it satisfies $1_{S} \triangleleft U^{* *} \cup U^{*}$ for all $U \in \operatorname{Pow}(S)$. Classically, a topological space is extremally disconnected iff its frame of open subsets is De Morgan [21. Obviously, $S$ Boolean implies $S$ De Morgan.

For $p \in \operatorname{Pow}(\{\top\})$, we shall suggestively write $P$ to stand for $\top \in p$, and ' $\forall p$ ' to mean 'for all $p$ in $\operatorname{Pow}(\{\top\})$ '. We set

$$
U_{P}=\left\{1_{S}: P\right\} \equiv\left\{1_{S}: \top \in p\right\} .
$$

Note that, in any formal space, $\left\{1_{S}\right\}^{*}={ }_{S} S^{*}={ }_{S} \emptyset$, and $\emptyset^{*}=S={ }_{S}\left\{1_{S}\right\}$.

Lemma 2.1 Let $S$ be any formal space.

i. If $(\forall p)(\exists x) x \in U_{P} \cup U_{P}^{*} \& \neg x \triangleleft \emptyset$, then $(\forall p) P \vee \neg P$, i.e. $(\forall p \in \operatorname{Pow}(\{\top\})) p \cup$ $p^{*}=\{\top\}$.

ii. If $(\forall p)(\exists x) x \in U_{P}^{* *} \cup U_{P}^{*} \& \neg x \triangleleft \emptyset$, then $(\forall p) \neg \neg P \vee \neg P$, i.e. $(\forall p \in \operatorname{Pow}(\{\top\}))$ $p^{* *} \cup p^{*}=\{\top\}$.

Proof. From $x \in U_{P}$ one gets $P$. On the other hand, from $x \in U_{P}^{*}$ and $\neg x \triangleleft \emptyset$ one obtains $\neg P$ as follows: assuming $P$, one has $U_{P}=\left\{1_{S}\right\}$, so that $U_{P}^{*} \triangleleft \emptyset$; together with $x \in U_{P}^{*}$ and $\neg x \triangleleft \emptyset$, this yields a contradiction, so that $\neg P$. Finally, assuming $\neg P$ gives $U_{P}=\emptyset$, and thus also $U_{P}^{* *}={ }_{S} \emptyset$; by $x \in U_{P}^{* *}, \neg x \triangleleft \emptyset$ one derives $\neg \neg P$. The reader may then easily fill in the details.

In [16] one finds an elegant 'arrow-theoretic' proof that no Boolean frame may have points, unless classical logic is accepted. Here is another formulation of that proof, and the corresponding fact for De Morgan locales:

Proposition 2.2 A Boolean (resp. De Morgan) formal space $S$ cannot have a point unless PEM, [R]EM in CZF (resp. DML, [R]DML in CZF), is accepted.

Proof. Assume $S$ has a point. 
$S$ Boolean implies $(\forall p) 1_{S} \triangleleft U_{P} \cup U_{P}^{*}$;

$S$ has a point $\alpha$ implies that $(\forall p)(\exists a) a \in U_{P} \cup U_{P}^{*} \& a \in \alpha$.

By $a \in \alpha$ one has $\neg a \triangleleft \emptyset$, and then one concludes using Lemma 2.1. The proof for the De Morgan case is similar.

As models of the settings in consideration (CZF, CTT, HHA, IZF) exist in which $\neg[R] D M L(\neg \mathrm{DML})$, is valid, in these settings De Morgan locales/formal spaces cannot be proved to have points. This implies that no such formal space is a topological space, i.e. no non-trivial Boolean or De Morgan frame can be obtained as the frame of opens of a non-empty (inhabited) topological space. Classically, of course, the lattice of open subsets of any discrete space is a Boolean frame with points.

Despite this result, at least in HHA/IZF, there are Boolean locales that are proper, i.e. such that, for all $U \in \operatorname{Pow}(S), S \triangleleft U$ implies $\exists a \in U$ (see [16]). Properness is a stronger formulation of non-triviality: a space is non-trivial if $\neg S \triangleleft$ $\emptyset$.

A formal space $(S, \triangleleft)$ is open (or overt, or has a positivity predicate [23, 32, 10]) iff there is a predicate $\operatorname{Pos}(x)$, for $x$ in $S$, satisfying

$$
\begin{array}{lr}
\text { i. } \operatorname{Pos}(a) \text { and } a \triangleleft U \text { imply }(\exists b \in S) b \in U \& \operatorname{Pos}(b) & \text { (monotonicity); } \\
\text { ii. } a \triangleleft U \text { implies } a \triangleleft U^{+} \equiv\{b \in U: \operatorname{Pos}(b)\} & \text { (positivity). }
\end{array}
$$

(Note that classically, all formal spaces are open, with $\operatorname{Pos}(a) \equiv \neg a \triangleleft \emptyset$ ). Then, although a Boolean locale can be proper, it cannot be open.

Proposition 2.3 No non-trivial De Morgan formal space $S$ (in particular, no nontrivial Boolean formal space) can be proved to be open in the considered intuitionistic settings.

Proof. Assume $S$ is open. Then, by positivity, $1_{S} \triangleleft\left\{1_{S}\right\}^{+}$. Assume $1_{S} \in\left\{1_{S}\right\}^{+}$, so that $\operatorname{Pos}\left(1_{S}\right)$ holds.

$S$ De Morgan implies $(\forall p) 1_{S} \triangleleft U_{P}^{* *} \cup U_{P}^{*}$;

by $\operatorname{Pos}\left(1_{S}\right)$ and monotonicity of Pos, for all $p$ there is $a \in U_{P}^{* *} \cup U_{P}^{*}$ with $\operatorname{Pos}(a)$.

Then, since $\operatorname{Pos}(a)$ implies $\neg a \triangleleft \emptyset$ (by monotonicity), by Lemma 2.1 one obtains $(\forall p) \neg \neg P \vee \neg P$. As CZF (various extensions thereof, CTT, and HHA) can be extended with the negation of (restricted) De Morgan law, one has that $1_{S} \in\left\{1_{S}\right\}^{+}$ 
leads to a contradiction in the extended setting, so that $\left\{1_{S}\right\}^{+}=\emptyset$. But this cannot be, as, by positivity $1_{S} \triangleleft\left\{1_{S}\right\}^{+}$, and we assumed the space to be non-trivial.

This proof shows that Boolean spaces can be proper only because the elements one extracts from each cover of the whole space are not required to be different from the bottom element, let alone positive.

Theorem 2.4 (GUP) Let $S$ be

i. a non-trivial Boolean formal space, or

ii. a non-trivial De Morgan formal space such that $(\forall x \in S) x \triangleleft \emptyset \vee \neg x \triangleleft \emptyset$;

then $S$ cannot be proved to be set-presented in type theory or in $C Z F+R E A+P A+S e p$.

Proof. $i$. Assume $S$ has a set-presentation. Then

$S$ Boolean implies: $(\forall p) 1_{S} \triangleleft U_{P} \cup U_{P}^{*}$;

$S$ set-presented implies: $(\forall p)\left(\exists i \in I\left(1_{S}\right)\right) C\left(1_{S}, i\right) \subseteq U_{P} \cup U_{P}^{*}$;

GUP implies: $\left(\exists i \in I\left(1_{S}\right)\right)(\forall p) C\left(1_{S}, i\right) \subseteq U_{P} \cup U_{P}^{*}$.

In particular, taking $p=\{\top\}$ so that $P=\top \in\{\top\}$ is true, this gives

(*) $\forall x \in C\left(1_{S}, i\right)\left(x=1_{S} \vee x \triangleleft \emptyset\right)$.

Then assume $x=1_{S} \in C\left(1_{S}, i\right)$. Since $C\left(1_{S}, i\right) \subseteq U_{P} \cup U_{P}^{*}$, and since $S$ is nontrivial, by Lemma 2.1, one gets $(\forall p) P \vee \neg P$. We saw that GUP is incompatible with the law of excluded middle. Therefore, by $(*)$, one gets $\forall x \in C\left(1_{S}, i\right) x \triangleleft \emptyset$; but $1_{S} \triangleleft C\left(1_{S}, i\right)$, and we assumed $S$ to be non-trivial, whence $S$ cannot be setpresented.

ii. Assume $S$ is set-presented. Note first that if $S$ satisfies $(\forall x \in S) x \triangleleft \emptyset \vee$ $\neg x \triangleleft \emptyset$, but has no top element $1_{S}$, also the isomorphic formal space with a top 12 will satisfy the given decidability condition, as, for the top element we have by hypothesis that $\neg 1_{S} \triangleleft \emptyset$. Then, using GUP one finds $i \in I\left(1_{S}\right)$ such that $(\forall p) C\left(1_{S}, i\right) \subseteq U_{P}^{* *} \cup U_{P}^{*}$. Let $x \in C\left(1_{S}, i\right)$, and assume $\neg x \triangleleft \emptyset$. By Lemma 2.1. one obtains $(\forall p) \neg \neg P \vee \neg P$. As this contradicts GUP, one has $\neg \neg x \triangleleft \emptyset$. By the decidability of $x \triangleleft \emptyset$, it follows that $x \triangleleft \emptyset$ for all $x \in C\left(1_{S}, i\right)$. But $S$ is non-trivial, so that it cannot be set-presented.

An example of a De Morgan non-Boolean formal space satisfying the condition in Theorem 2.4 is presented in the next section.

This theorem shows that the formal spaces that cannot be inductively generated consist not just of few pathological cases. In particular, one has: 
Corollary 2.5 Given any non-trivial formal space $S$, the formal space $S^{* *} \equiv(S$, $\left.\triangleleft^{* *}\right)$, where $a \triangleleft^{* *} U \Longleftrightarrow\{a\}^{* *} \triangleleft U^{* *}$, can not be proved set-presented in type theory, $C Z F+R E A+P A+S e p$.

The space $S^{* *}$ is indeed the Boolean formal subspace corresponding to the $* *$-nucleus on the frame defined by $S$, i.e. the space associated with the frame of 'regular' elements of $\operatorname{Sat}(S)$ (see e.g. [16, 21] for a discussion of the $* *$-nucleus on a locale $L$ ) 3 This is non-trivial as soon as $S$ is non-trivial.

These subspaces/nuclei are, also classically, a peculiarity of locale theory (as opposed to point-set topology), since, given any locale $L$, the $* *-$ nucleus on $L$ yields the least dense sublocale of $L$. (This need not exist in a topological space; consider e.g. the real line: the rationals and the irrationals define dense disjoint subspaces).

The following proposition shows that compactness too conflicts with being Boolean:

Proposition 2.6 No non-trivial Boolean formal space $S$ can be compact unless PEM ([R]EM) is accepted.

Proof. Assume $S$ is compact.

$S$ Boolean implies $(\forall p) 1_{S} \triangleleft U_{P} \cup U_{P}^{*}$;

$S$ compact implies that $1_{S} \triangleleft u_{0} \subseteq U_{P} \cup U_{P}^{*}$, with $u_{0}$ finite.

It is a standard fact that $u_{0} \subseteq V \cup W$, with $u_{0}$ finite, implies intuitionistically $u_{0}=$ $v_{0} \cup w_{0}$, with $v_{0} \subseteq V, w_{0} \subseteq W$ both finite [6]. Thus we have finite $v_{0} \subseteq U_{P}, w_{0} \subseteq U_{P}^{*}$, with $1_{S} \triangleleft v_{0} \cup w_{0}$; moreover, 'finite' implies 'either empty or inhabited'. By cases: $v_{0}, w_{0}=\emptyset$ cannot be, by non-triviality. Then one of the following alternatives holds:

1. $v_{0}, w_{0}$ inhabited, or

2. $v_{0}$ inhabited and $w_{0}=\emptyset$, or

3. $v_{0}=\emptyset$ and $w_{0}$ inhabited.

The first and second case directly give $P \vee \neg P$. For the last, assuming $P$ one gets $w_{0} \triangleleft \emptyset$, that together with $v_{0}=\emptyset$, gives $1_{S} \triangleleft \emptyset$, so that, by non-triviality of $S, \neg P$. Therefore, if $S$ is compact, the law $(\forall p) P \vee \neg P$ holds.

\footnotetext{
${ }^{3}$ In the literature on locales this nucleus is also known as the Booleanization of $S$. There are many Boolean sublocales of a given locale $L$, but each of them can be seen as defined by a $* *-$ nucleus over a closed sublocale of $L$ [16].
} 
In $\mathrm{CZF}$, or CTT, more generally, no non-trivial Boolean space $S$ can be proved to be locally compact, since locally compact formal spaces are set-presented [1], and by Theorem 2.4 no non-trivial Boolean space $S$ can be proved to be set-presented ('more generally': any Boolean $S$ is regular, and a compact regular locale is locally compact, e.g. 21]). Classically (e.g. in ZF), every finite discrete space has a compact Boolean frame of opens.

So far the generalized uniformity principle has only been used to show that a constructive system cannot prove that formal spaces of a certain type can be setpresented. We conclude this section with two other important consequences of the consistency of CZF, CTT with this principle. Contrary to what one may expect, in $H H A$ or IZF, De Morgan locales can be compact: the classical result (due to Stone) that the (compact) frame $\operatorname{Idl}(B)$ of ideals over a Boolean algebra $B$ is De Morgan if and only if $B$ is complete (e.g. [21]) is topos-valid. The following is one half of this result, formulated for formal spaces. Recall that in fully impredicative settings, frames and set-generated class-frames come to the same thing, so that $\operatorname{Sat}(S)$ is carried by a set for every space $S$.

Proposition 2.7 (Stone) Assume $S$ is a Boolean formal space such that its frame of opens $\operatorname{Sat}(S)$ is a set. Then the formal space $S_{\beta} \equiv\left(\operatorname{Sat}(S), \triangleleft_{\beta}\right)$, with $U \triangleleft_{\beta}$ $\left\{U_{i}\right\}_{i \in I} \Longleftrightarrow U \triangleleft U_{i_{1}} \cup \ldots \cup U_{i_{n}}$ for $\left\{i_{1}, \ldots, i_{n}\right\}$ a (possibly empty) finite subset of $I$, is a compact De Morgan formal space.

Proof. The proof that $S_{\beta}$ is a formal space is left to the reader. One has to prove

$$
S \triangleleft_{\beta}\left(\left\{U_{i}\right\}_{i \in I}\right)^{*} \cup\left(\left\{U_{i}\right\}_{i \in I}\right)^{* *}
$$

for any given set $\left\{U_{i}\right\}_{i \in I}$ of elements of $\operatorname{Sat}(S)$. Routine calculations show that

$$
\left(\left\{U_{i}\right\}_{i \in I}\right)^{*}={ }_{\beta}\left\{\left(\bigcup_{i \in I} U_{i}\right)^{* S}\right\}
$$

$\left({ }^{*}\right.$ is pseudo-complementation in $\left.S\right)$, so that

$$
\left(\left(\left\{U_{i}\right\}_{i \in I}\right)^{*}\right)^{*}=\beta\left\{\left(\bigcup_{i \in I} U_{i}\right)^{*_{S} *_{S}}\right\} .
$$

Since $S$ is Boolean, $\left(\bigcup_{i \in I} U_{i}\right)^{*_{S} *_{S}}={ }_{S} \bigcup_{i \in I} U_{i}$. As

$$
S \triangleleft\left(\bigcup_{i \in I} U_{i}\right)^{*_{S}} \cup\left(\bigcup_{i \in I} U_{i}\right)={ }_{S}\left(\bigcup_{i \in I} U_{i}\right)^{*_{S}} \cup\left(\bigcup_{i \in I} U_{i}\right)^{*_{S} *_{S}},
$$

one has $S \triangleleft_{\beta}\left\{\left(\bigcup_{i \in I} U_{i}\right)^{*_{S}},\left(\bigcup_{i \in I} U_{i}\right)^{*_{S} *_{S}}\right\}$ by definition of $\triangleleft_{\beta}$ (note that pseudocomplements are saturated). Therefore, by 1,2 above, $S \triangleleft_{\beta}\left(\left\{U_{i}\right\}_{i \in I}\right)^{*} \cup\left(\left\{U_{i}\right\}_{i \in I}\right)^{* *}$, as wished.

Despite this fact, one has: 
Proposition 2.8 No non-trivial De Morgan formal space $S$ such that $(\forall x \in S)$ $\neg \neg x \triangleleft \emptyset \vee \neg x \triangleleft \emptyset$ is compact unless [R]DML (DML) holds.

Proof. Assume $S$ is compact. For all $p \in \operatorname{Pow}(\{\top\})$, one finds a finite $v_{0}$ with $v_{0} \subseteq$ $U_{P}^{* *} \cup U_{P}^{*}, 1_{S} \triangleleft v_{0}$. One has, in particular, $\left(\forall x \in v_{0}\right) \neg \neg x \triangleleft \emptyset \vee \neg x \triangleleft \emptyset$. By a general intuitionistic principle (see [23, Lemma 2.4]), this gives $\left(\forall x \in v_{0}\right)(\neg \neg x \triangleleft \emptyset) \vee(\exists x \in$ $\left.v_{0}\right)(\neg x \triangleleft \emptyset)$. Since $v_{0}$ is finite, we get $\neg \neg\left(\forall x \in v_{0}\right)(x \triangleleft \emptyset) \vee\left(\exists x \in v_{0}\right)(\neg x \triangleleft \emptyset)$. It cannot be that $x \triangleleft \emptyset$ for all $x \in v_{0}$. Thus, there is $x \in v_{0}$ with $\neg x \triangleleft \emptyset$, so that one concludes by Lemma 2.1 .

As a consequence, in a topos that does not satisfy De Morgan law, no frame of the form $\operatorname{Idl}(B)$, with $B$ complete Boolean algebra, can have a base satisfying the decidability condition in the above proposition. Note also that such frames are examples of De Morgan non-Boolean frames, given that no Boolean frame can be proved compact.

Using the generalized uniformity principle, the above proposition may be strengthened:

Theorem 2.9 (GUP) No non-trivial De Morgan formal space $S$ can be proved to be compact in type theory, $C Z F+R E A+P A+S e p$.

Proof. Using GUP, one has that a finite subset $u_{0}=\left\{x_{1}, \ldots, x_{n}\right\}$ of $S$ exists such that $(\forall p) 1_{S} \triangleleft u_{0} \subseteq U_{P}^{*} \cup U_{P}^{* *}$ ( $u_{0}$ is non-empty, as $S$ is non-trivial). Assume $\neg x_{1} \triangleleft \emptyset \vee \ldots \vee \neg x_{n} \triangleleft \emptyset$. By Lemma 2.1, one has that $(\forall p) \neg \neg P \vee \neg P$ holds. We saw that this principle is incompatible with GUP, so that $\neg\left(\neg x_{1} \triangleleft \emptyset \vee \ldots \vee \neg x_{n} \triangleleft \emptyset\right)$. This gives $\neg \neg x_{1} \triangleleft \emptyset \& \ldots \& \neg \neg x_{n} \triangleleft \emptyset$, that is $\neg \neg\left(x_{1} \triangleleft \emptyset \& \ldots \& x_{n} \triangleleft \emptyset\right)$. On the other hand, from $1_{S} \triangleleft u_{0}$ one gets $\neg\left(x_{1} \triangleleft \emptyset \& \ldots \& x_{n} \triangleleft \emptyset\right)$, so that $S$ cannot be compact.

By this result it follows that no non-trivial frame can be assumed to be carried by a set in a constructive setting (see [13] for a more direct proof):

Corollary 2.10 (GUP) Every non-trivial frame Sat $(S)$, for $S$ Boolean, is carried by a proper class in type theory, $C Z F+R E A+P A+S e p$. Thus, no non-trivial frame Sat $(S)$ may be proved to have a set of elements in these contexts.

Proof. If the collection of elements of $\operatorname{Sat}(S)$ could be proved to be constructively a set, by Proposition 2.7 the formal space $S_{\beta}$ would be compact and De Morgan, contradicting Theorem 2.9. Now assume a frame $S a t(S)$ has a set of elements; then all frames $S a t\left(S^{\prime}\right)$, for $S^{\prime}$ a subspace of $S$, are carried by a set, too, so that also $\operatorname{Sat}\left(S^{* *}\right)$ should be. 
Remarks. $i$. The property of Boolean formal spaces that has been exploited in the proofs in this section is that the whole space $S$ is covered by $U_{P} \cup U_{P}^{*}$, for all $p$ in $\operatorname{Pow}(\{\top\})$. It is easy to check that a morphism $f: S \rightarrow S^{\prime}$, with $S$ Boolean, preserves pseudocomplements. It follows that whenever such a morphism exists, one also has $(\forall P) 1_{S^{\prime}} \triangleleft V_{P} \cup V_{P}^{*}$, with $V_{P} \equiv\left\{1_{S^{\prime}}: P\right\}$. Then, Propositions 2.2, 2.3, 2.6, and Theorem 2.4 hold true more generally if one replaces $S$ with any non-trivial codomain of a morphism with Boolean domain. Similar considerations also hold in connection with the results concerning De Morgan spaces, when $f: S \rightarrow S^{\prime}$ is any morphism that preserves pseudocomplements (in particular, when $f$ defines an open continuous functions of locales/formal spaces [23]).

ii. Though not set-presentable, Boolean formal spaces are constructively useful: an example of the use of a Boolean formal space to obtain a concrete (constructive and predicative) description of ideal non-effective objects can for instance be found in [7, Theorem 6.1].

iii. In contrast with the Boolean case, the class of De Morgan formal spaces is not closed for subspaces: an extremally disconnected space may have Hausdorff subspaces that are not extremally disconnected; classically, but this suffices. In [20], the following law is considered: for all propositions $P, Q$

$$
(P \rightarrow Q) \vee(Q \rightarrow P)
$$

This principle is stronger than De Morgan's (take $Q$ to be $\neg P$ ), and is inherited by the internal logic of sheaf subtoposes [20. Call strongly De Morgan a formal space such that the associated frame models this formula. A strongly De Morgan formal space is De Morgan. It is easy to prove that the class of strongly De Morgan formal spaces is closed for subspaces.

\section{Dedekind-MacNeille completions}

Given a set $S$, and any (class-)relation $R(a, U)$, for $a \in S$ and $U \in \operatorname{Pow}(S)$, one may define $R$ to be set-presented precisely as for coverings. Let $\Phi(P)$ be an instance of a law in one variable $P$ that is incompatible with GUP, e.g. $\Phi(P) \equiv P \vee \neg P$ (in $\mathrm{CZF}, p \cup p^{*}=\{\top\}$, for $\left.p \in \operatorname{Pow}(\{\top\})\right)$.

Proposition 3.1 (GUP) Let $R \subseteq S \times \operatorname{Pow}(S)$, for $S$ set. Assume that, for some $a$ in $S$, one has $\neg R(a, \emptyset)$, and $(\forall p) R\left(a, U_{\Phi(P)}\right)$, where $U_{\Phi(P)} \equiv\{a: \Phi(P)\}$. Then $R$ cannot be proved to be set-presented in type theory, $C Z F+R E A+P A+S e p$.

Proof. Assume $R$ is set-presented by $C(x, i)(x \in S, i \in I(x))$. By GUP, there is $i \in I(a)$ such that $(\forall p) C(a, i) \subseteq U_{\Phi(P)}$ and $R(a, C(a, i))$. Assume $x \in C(a, i)$. Then 
$(\forall p) \Phi(P)$. By hypothesis this contradicts GUP. Therefore, $C(a, i)=\emptyset$, and $R(a, \emptyset)$, against what we have assumed.

Recall that the Dedekind-MacNeille completion of a partial order makes it possible to embed a given partial order in a complete lattice preserving meets and joins that exist (see e.g. [33, 35]). Given a partially ordered set $(S, \leq)$, one may define a relation $R_{c}(x, U)$ by letting

$$
R_{c}(x, U) \Longleftrightarrow(\forall y)[(\forall u \in U) u \leq y] \rightarrow x \leq y \Longleftrightarrow x \in \bigcap_{U \subseteq \downarrow y} \downarrow y .
$$

To have that $R_{c}$ is a covering relation, the Dedekind-MacNeille covering, the partial order has to satisfy some further conditions. In particular, if $S$ is a Heyting algebra this is always the case. The frame $\operatorname{Sat}\left(S, R_{c}\right)$ of saturated subsets of the formal topology $\left(S, R_{c}\right)$ is then the complete lattice in which the Heyting algebra $S$ is embedded via $e: S \rightarrow S a t\left(S, R_{c}\right), e(a)=\mathcal{S}(\{a\})$. Recall that, as over any frame, an implication operation making $S a t\left(S, R_{c}\right)$ a complete Heyting algebra can be defined by letting $U \rightarrow V \equiv\{a \in S: a \downarrow U \triangleleft V\}$. The Heyting algebra structure of $\operatorname{Sat}\left(S, R_{c}\right)$ then extends that of $S$ (see e.g. [35]).

T. Coquand has suggested that no Dedekind-MacNeille covering can be constructively proved to be set-presented (see also 9]). We prove here that this holds for every relation $R_{c}(x, U)$ on a given poset $(S, \leq)$, but with a further hypothesis.

Proposition 3.2 (GUP) Let $(S, \leq)$ be a partial order with at least two elements $a, b$ such that $\neg a \leq b$, and such that $a$ is "stable", i.e. a satisfies: $\neg \neg a \leq x$ implies $a \leq x$, for all $x$. Then the relation $R_{c}(x, U)$ cannot be proved to be set-presented in type theory, $C Z F+R E A+P A+S e p$.

Proof. By Proposition 3.1, it suffices to show that $\neg R_{c}(a, \emptyset)$ and $(\forall p) R_{c}\left(a, U_{\Phi(P)}\right)$, with $U_{\Phi(P)} \equiv\{a: \Phi(P)\}$, and $\Phi(P) \equiv P \vee \neg P$. If $R_{c}(a, \emptyset)$, then $a \in \bigcap_{\emptyset \subseteq \downarrow y} \downarrow y$. As $\emptyset \subseteq \downarrow b$, we get $a \leq b$, against the hypothesis. For the second, let $U_{\Phi(P)} \subseteq \downarrow y$, and assume $\neg a \leq y$ and $\Phi(P)$. Then $U_{\Phi(P)}=\{a\} \subseteq \downarrow y$, so that $a \leq y$, against what we have assumed. This gives $\neg \Phi(P)$. As $\neg \neg \Phi(P)$ is intuitionistically provable, we get $\neg \neg a \leq y$, whence $a \leq y$. We conclude that $a \in \bigcap_{U_{\Phi(P)} \subseteq \downarrow} \downarrow y$, for all $p$, i.e. $(\forall p) R_{c}\left(a, U_{\Phi(P)}\right)$.

Note that this proof is, in essence, a simplification and a generalization of the proof for the special case considered in [10] (see also below).

In particular, any poset with at least two elements and a decidable order relation satisfies the indicated requirements.

\footnotetext{
${ }^{4}$ On the occasion of the presentation of the material in the preceding sections at the workshop "Trends in constructive mathematics", Chiemsee (Germany) June 19-23, 2006.
} 
This proposition may be used to produce examples of non-De Morgan formal spaces that cannot be constructively set-presented.

Corollary 3.3 (GUP) Let $H \equiv(S, \wedge, \vee, \rightarrow, 0,1)$ be a Heyting algebra, with $S$ set containing elements $a, b$ satisfying the hypotheses in Proposition 3.2. Assume in $H$ De Morgan law is false, i.e., there is $c \in S$ such that $1 \neq c^{*} \vee c^{* *}$. Then the relation $R_{c}(x, U)$, defining the Dedekind-MacNeille cover on $H$, defines a nonDe Morgan formal space that cannot be proved to be set-presented in type theory, $C Z F+R E A+P A+S e p$.

Proof. As already recalled, the (set-generated) frame $\operatorname{Sat}\left(S, R_{c}\right)$ associated with the Dedekind-MacNeille cover defined over an Heyting algebra $H$ is a complete Heyting algebra in which the Heyting algebra operations extend the corresponding operations on $H$. As $a^{*}=a \rightarrow 0$ one can conclude.

The set $T=\left\{0, \frac{1}{2}, 1\right\}$ endowed with the natural order is a non-Boolean Heyting algebra. The Dedekind-MacNeille cover over this poset defines a De Morgan nonBoolean formal space $\left(T, \triangleleft_{D M}\right)$. That $T$ is non-Boolean again follows by the fact that the complete Heyting algebra $\operatorname{Sat}(T)$ is such that the Heyting algebra operations are extensions of the corresponding operations of $T$. To prove that $\left(T, \triangleleft_{D M}\right)$ is De Morgan, i.e. that $1 \in \bigcap K$, with $K \equiv\left\{\downarrow y: U^{*} \cup U^{* *} \subseteq \downarrow y\right\}$, for every $U \in \operatorname{Pow}(T)$, first one notes that $U^{*} \cup U^{* *} \subseteq \downarrow 1$; unwinding the definitions, one then proves that assuming $1 \notin U^{*} \cup U^{* *}$ leads to a contradiction, so that $\neg 1 \notin U^{*} \cup U^{* *}$. Therefore, $\downarrow \frac{1}{2} \notin K$ and $\downarrow 0 \notin K$, whence $1 \in \bigcap K$. By Proposition 3.2 (or Theorem 2.4) one has that $\left(T, \triangleleft_{D M}\right)$ is not constructively set-presentable.

Remark. If in the hypotheses of Proposition [3.1, $R$ is a covering $\triangleleft$ on a set $S$, and if $a \equiv 1_{S}={ }_{S} S$, then for every morphism $f: S \rightarrow S^{\prime}$, by $1_{s} \triangleleft U_{\Phi(P)}$ one gets $1_{S^{\prime}} \triangleleft U_{\Phi(P)}^{\prime}$, with $U_{\Phi(P)}^{\prime} \equiv\left\{1_{S^{\prime}}: \Phi(P)\right\}$. Therefore, if $S^{\prime}$ is non-trivial, by Proposition 3.1 it is not set-presentable. As an immediate corollary one has in particular that no formal space defined by the Dedekind-MacNeille cover on a poset $(S, \leq)$ with the properties in Proposition 3.2 , and such that $a$ is also the greatest element of $(S, \leq)$, may have points (as points are in a bijective correspondence with morphisms from the given topology to the set-presentable topology $\operatorname{Pow}(\{\top\}))$, and that every non-trivial formal subspace of $S$ is not set-presentable (if $S^{\prime} \equiv\left(S, \triangleleft^{\prime}\right)$ is a subspace of $S$, letting $e(a)=\{a\}$ for all $a \in S$ defines a morphism $\left.e: S \rightarrow S^{\prime}\right)$.

In fact, by the proof that $\neg R_{c}(a, \emptyset)$ and $R_{c}\left(a, U_{\Phi(P)}\right)$ for all $P$, with $\Phi(P) \equiv$ $\left(p \cup p^{*}=\{\top\}\right)$ (cf. proof of Proposition 3.2), the next result follows immediately without any reference to the uniformity principle.

Proposition 3.4 Let $S$ be a Dedekind-MacNeille topology defined on a poset with the properties in Proposition 3.2, and having a as greatest element. If $S$ has a point, 
or is compact, then PEM ([R]EM) holds. Furthermore, $S$ cannot be proved to be open in the intuitionistic settings considered.

We conclude this section with a discussion of Open Problem 4.5 of [10. Taking $S=\{0,1\}$ with the natural order, and $a \triangleleft U \equiv R_{c}(a, U)$ we obtain the formal space that is in [10] shown not to be set-presentable in CTT. Let us denote this space by $S_{D M}$.

We already pointed out that a uniform method for the definition of products of arbitrary formal spaces is generally regarded as being beyond constructive means. Open Problem 4.5 of [10] asked whether at least the particular product of $S_{D M}$ with itself is predicatively definable. The answer in this (indeed very special) case is yes. We show this with a slight detour: the 'double negation' formal topology $\operatorname{Pow}(\{\top\})_{\neg \neg}$, is defined by

$$
S=\{\top\}, \top \triangleleft U \Longleftrightarrow \neg \neg \top \in U .
$$

In [17, 18] it is shown that the system CZF cannot prove Pow $(\{\top\}) \neg \neg$ to be setpresentable (see also [19]). Note that $\operatorname{Pow}(\{\top\}) \neg \neg$ is isomorphic with the Boolean formal space $\operatorname{Pow}(\{T\})^{* *}$ of regular elements of $\operatorname{Pow}(\{\top\})$ (cf. section 2).

In fact it is easy to see that $\operatorname{Pow}(\{\top\})_{\neg \neg}$ and $S_{D M}$ are the 'same' formal space.

Lemma 3.5 $S_{D M} \cong \operatorname{Pow}(\{\top\})_{\neg\urcorner}$.

Proof. It is an exercise in intuitionistic logic to prove that $0={ }_{S_{D M}} \emptyset, \neg \neg 1 \in$ $U \Longleftrightarrow 1 \triangleleft_{D M} U$, and $\neg \neg \top \in U$ implies $1 \triangleleft_{D M}\{1: \top \in U\}$. It follows that the homomorphisms $f: S_{D M} \rightarrow \operatorname{Pow}(\{\top\})_{\neg}$ and $g: \operatorname{Pow}(\{\top\})_{\neg \neg} \rightarrow S_{D M}$, given by $f(0)=\emptyset, f(1)=\{\top\}$, and $g(\top)=\{1\}$, yield the required isomorphism.

Now the product $\operatorname{Pow}(\{\top\})_{\neg \neg} \times \operatorname{Pow}(\{\top\})_{\neg \neg}$ in FSp is simply $\operatorname{Pow}(\{\top\})_{\neg \neg}$ itself: $\operatorname{Pow}(\{\top\})_{\neg \neg}$ is 'almost' a terminal object in FSp, if a morphism with $\operatorname{Pow}(\{\top\})_{\neg \neg}$ as domain exists, then it is unique.

In 14] a general solution is provided to the problem of defining the product (and more generally, limits of arbitrary diagrams) of formal spaces by embedding the category of formal spaces in a complete category, thus regarding products as being potentially objects of a wider category.

\section{Appendix: The generalized uniformity principle in type theory}

In the type-theoretic context the generalized uniformity principle reads informally as follows: given any set $I$, and any mapping $R$ into the type of propositions $P R O P$ taking a proposition and an element of $I$ as arguments, if a mapping $F$ is given from $P R O P$ to $I$ such that $R(P, F(P))$ holds for all $P$, then one may find an element 
$\bar{i} \in I$ such that $R(P, \bar{i})$ holds for all $P$. We denote this version of the principle by GUP-CTT. Observe that, due to the propositions-as-sets identification, the type $P R O P$ of propositions may be replaced in GUP-CTT by the type $S E T$ of sets. In [10] this principle is formulated implicitly and it is claimed that GUP-CTT can be 'added' consistently to type theory. Models, due to T. Coquand, of type theory validating this form of the uniformity principle have then been discussed in [30].

Formally, GUP-CTT can be expressed in type theory (more specifically, in the logical framework $[29,8]$ ) by the addition of two constants UP1, UP2 as follows (cf. [30]):

$U P 1:(I: S E T) \rightarrow R:(P R O P \rightarrow E l(I) \rightarrow P R O P) \rightarrow F: P R O P \rightarrow E l(I) \rightarrow$ $(P: P R O P \rightarrow \operatorname{El}(R(P, F(P)))) \rightarrow \operatorname{El}(I) ;$

$U P 2:(I: S E T) \rightarrow R:(P R O P \rightarrow E l(I) \rightarrow P R O P) \rightarrow F: P R O P \rightarrow E l(I) \rightarrow$ $(G:(P: P R O P) \rightarrow \operatorname{El}(R(P, F(P)))) \rightarrow(P: P R O P) \rightarrow \operatorname{El}(R(P, U P 1(I R F G)))$.

Acknowledgments. I began the research described in this note while visiting the university of Birmingham in 2005. Thanks go to the "Fondazione Gini" for financially supporting that visit. In that occasion I enjoyed stimulating conversations with Steven Vickers on this and related topics.

I am grateful to Thierry Coquand, Milly Maietti, and Pino Rosolini for a helpful and interesting e-mail discussion on the consistency of the generalized uniformity principle with type theory, and to Thomas Streicher for letting me know about the proof of the consistency of this principle with CZF+REA+PA+Sep in Benno van den Berg's thesis. Thanks also go to Peter Aczel for his useful comments on a previous draft of this note.

\section{References}

[1] P. Aczel, "Aspects of general topology in constructive set theory". Ann. Pure Appl. Logic, 137 (2006), no. 1-3, 3-29.

[2] P. Aczel, G. Curi, "On the $T_{1}$ axiom and other separation properties in constructive point-free and point-set topology". Ann. Pure Appl. Logic. To appear. [doi:10.1016/j.apal.2009.03.005]

[3] P. Aczel, M. Rathjen, "Notes on Constructive Set Theory", Mittag-Leffler Technical Report No.40, 2000/2001.

[4] B. van den Berg, Predicative topos theory and models for constructive set theory. $\mathrm{PhD}$ thesis, University of Utrecht (2006).

[5] B. van den Berg, I. Moerdijk, "Aspects of predicative algebraic set theory II: realizability". Theor. Comp. Science. To appear. [Available from: http://arxiv.org/abs/0801.2305. 
[6] T. Coquand, "An intuitionistic proof of Tychonoff theorem", J. Symb. Log., vol. 57, n. 1 (1992), pp. 28-32.

[7] T. Coquand, H. Lombardi, "A logical approach to abstract algebra. A survey". Math. Struct. in Comput. Science 16, (2006), pp. 885-900.

[8] T. Coquand, P. Dybjer, E. Palmgren, A. Setzer, Type-theoretic foundation of constructive mathematics. In preparation.

[9] T. Coquand, S. Sadocco, G. Sambin, J. Smith, "Formal topologies on the set of first-order formulae". J. Symb. Log., Vol. 65, 3 (2000), pp. 1183-1192.

[10] T. Coquand, G. Sambin, J. Smith, S. Valentini, "Inductively generated formal topologies". Ann. Pure Appl. Logic (2003), 124, no. 1-3, pp. 71-106.

[11] G. Curi, Geometry of Observations. Some contributions to (constructive) point-free topology. Ph.D. Thesis, University of Siena (2003).

[12] G. Curi, "Exact approximations to Stone-Čech compactification". Ann. Pure Appl. Logic, 146, 2-3, 2007, pp. 103-123.

[13] G. Curi, "On the existence of Stone-Čech compactification". Submitted.

[14] G. Curi, "The category of frame presentations". Forthcoming.

[15] M. Fourman, R. Grayson, "Formal Spaces". In The L.E.J. Brouwer Centenary Symposium, A.S. Troelstra and D. van Dalen, eds. North Holland (1982), pp. 107122.

[16] M. Fourman, D.S. Scott, "Sheaves and logic". In Applications of sheaves, M. Fourman et al. eds., Springer LNM 753, Springer-Verlag, 1979, pp. 302-401.

[17] N. Gambino, "Sheaf interpretations for generalized predicative intuitionistic systems", Ph.D. Thesis, University of Manchester (2002).

[18] N. Gambino, "Heyting-valued interpretations for Constructive Set Theory", Ann. Pure Appl. Logic, 137, 1-3, 2006, pp. 164-188.

[19] R.J. Grayson, "Forcing in intuitionistic systems without powerset", J. Symb. Log. 48 (1983) pp. 670-682.

[20] P. T. Johnstone, "Conditions related to De Morgan's law". In Applications of sheaves, M. Fourman et al. eds., Springer LNM 753, Springer-Verlag, 1979, pp. 479-491.

[21] P. T. Johnstone, Stone Spaces. Cambridge University Press, 1982.

[22] P. T. Johnstone, "The points of pointless topology", Bulletin of the American Mathematical Society, Vol. 8, Number 1, January 1983.

[23] P. T. Johnstone, "Open locales and exponentiation". Contemp. Math., 30 (1984), pp. 84-116. 
[24] P. T. Johnstone, "The art of pointless thinking: a student's guide to the category of locales". In Category theory at work, H. Herrlich and H. Porst eds, Helderman Verlag, 1991, pp. 85-107.

[25] P. Martin-Löf, Intuitionistic Type Theory. Notes by G. Sambin. Studies in Proof Theory. Bibliopolis, Napoli (1984).

[26] E. Palmgren, "Predicativity problems in point-free topology". In: V. StoltenbergHansen et al. eds. Proceedings of the Annual European Summer Meeting of the Association for Symbolic Logic, held in Helsinki, Finland, August 14-20, 2003, Lecture Notes in Logic 24, ASL, AK Peters Ltd, 2006.

[27] R.S. Lubarsky, "CZF and Second Order Arithmetic". Accepted for publication in Ann. Pure Appl. Logic.

[28] S. MacLane, I. Moerdijk, Sheaves in Geometry and Logic - A First Introduction to Topos Theory. Springer, 1992.

[29] B. Nördstrom, K. Peterson, J. Smith, Programming in Martin-Löf's Type Theory (Clarendon Press), Oxford, 1990.

[30] B. Petit, "Polymorphic type theory and Uniformity Principle". Preprint.

[31] G. Rosolini, "About modest sets". Int. J. Found. Comp. Sci. 1 (1990) pp. 341-353.

[32] G. Sambin, "Intuitionistic formal spaces - a first communication", in Mathematical Logic and its Applications, D. Skordev, ed. Plenum, 1987, pp. 187-204.

[33] G. Sambin, "Pretopologies and completeness proofs". J. Symb. Log. 60, No.3, 861878 (1995).

[34] T. Streicher, "Realizability models for CZF $+\neg$ Pow". Unpublished note.

[35] A. Troelstra, D. van Dalen, Constructivism in mathematics, an introduction. Volume I. Studies in logic and the foundation of mathematics, vol. 121, North-Holland. 